\title{
BCH codes in UFMC: A new contender candidate for 5G communication systems
}

\author{
Ghasan Ali Hussain ${ }^{1}$, Lukman Audah² \\ ${ }^{1}$ Department of Electrical Engineering, Faculty of Engineering, University of Kufa, Iraq \\ ${ }^{2}$ Wireless and Radio Science Centre (WARAS), Faculty of Electrical and Electronic Engineering, Universiti Tun Hussein \\ Onn Malaysia, Johor, Malaysia
}

\begin{tabular}{l} 
Article Info \\
\hline Article history: \\
Received Jul 20, 2020 \\
Revised Nov 25, 2020 \\
Accepted Dec 12, 2020 \\
\hline
\end{tabular}

\section{Keywords:}

$5 \mathrm{G}$

BCH Codes

f-OFDM

OFDM

UFMC

\begin{abstract}
Nowadays, fifth generation (5G) wireless network is considered one of the most important research topics in wireless industry and it will be substituting with fourth generation (4G) in several aspects. Although the robustness of orthogonal frequency division multiplexing (OFDM) system against channel delays which is the reason behind using it in LTE/LTE Advanced however, it is suffering from high peak to average power ration (PAPR) and out of band side lobes. So, universal filtered multi-carrier (UFMC) technique is considered a new modulation scheme for $5 \mathrm{G}$ wireless communication system to overcome on the common OFDM demits. In contrast, to achieve reliable data transmission in digital communication systems, using error correcting codes are considered an essential over noisy channels. In this paper, BCH code has been used for UFMC system over AWGN. The results showed that using $\mathrm{BCH}$ codes in UFMC contributed in enhancing BER performance while could decreasing both of PAPR and OOBE values better than conventional OFDM system.
\end{abstract}

This is an open access article under the CC BY-SA license.

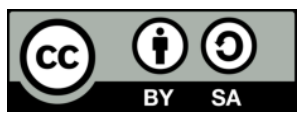

\section{Corresponding Author:}

Lukman Audah

Wireless and Radio Science Centre (WARAS), Faculty of Electrical and Electronic Engineering

Universiti Tun Hussein Onn Malaysia

Parit Raja, 86400 Batu Pahat, Johor, Malaysia

Email: hanif@uthm.edu.my

\section{INTRODUCTION}

Nowadays, fifth generation $(5 \mathrm{G})$ wireless network is considered one of the most important research topic in wireless industry and it will be substituting with fourth generation $(4 \mathrm{G})$ in several aspects, owing to its potential applications in the evolution of new applications; like vehicular communications, internet of things (IoT) and low latency and ultra-reliable communications [1,2]. Whilst, 5G wireless technologies which characterized of low power consumption and high spectral efficiency expected to introduced in 2020 and beyond [3, 4]. Comparing to present communication systems, 5G could achieve higher speed, better reliability and more security for each of machines, human users and man to machine interactions [5]. Thus, 5G cellular communications have been attracted a lots of attentions in both of industrial and academic fields [6]. The main core technique of LTE standard was orthogonal frequency division multiplexing (OFDM), nevertheless it could not be a promising solution for $5 \mathrm{G}$ and future wireless communications due to its disadvantages. Where, high spectrum side lobes are considered one of the drawbacks of OFDM which is happening because of the rectangular pulses of its symbols whilst, OFDM are suffering from the inability of supporting a significant feature namely flexible numerology [7]. 
Although the robustness of OFDM system against channel delays which is the reason behind using it in LTE/LTE Advanced however, it is suffering from high peak to average power ration (PAPR) which is caused of increasing the consumption of battery and lower efficiency of power amplifier, while low spectrum efficiency is happening due to high out of band side lobes in OFDM system. Thus, new modulation technologies have been required for $5 \mathrm{G}$ communication systems which are characterized of the ability of overcome of OFDM disadvantages [8]. Where, filtered-OFDM (f-OFDM) and universal filtered multi-carrier (UFMC) techniques have been proposed in [8-11] as new modulation schemes for 5G systems to overcome on the common OFDM demits.

In other hand, different waveforms candidates have been suggested for 5G in [12] such as f-OFDM, UFMC, generalized frequency division multiplexing (GFDM) and filter bank multicarrier (FBMC). They were assessing these waveforms under key performance indicators factors (PAPR, filter length, computational complexity, spectral efficiency and latency). They concluded that both of UFMC and FBMC are better coexistence with CP-OFDM that used in 4G networks. Whereas, both of UFMC and f-OFDM were more flexibility than GFDM and FBMC in reducing the complexity. While, all these waveforms candidates have lower PAPR than OFDM which is maybe results due to its utilizing filter and windowing techniques [12]. In contrast, the spectrum utilization of UFMC has been described in [13]. Where, several modulation techniques have been also reviewed and motivated the needing of UFMC in 5G wireless communication. They concluded that spectrum utilization of UFMC was much better than OFDM system and thus, UFMC is considered a right choice as candidate for $5 \mathrm{G}$.

In wireless communication systems, one of the aims of next generation are introducing high data rate applications and service like, data and image in local coverage networks, video streaming, web browsing, wireless teleconferencing and multimedia. Quality digital communication systems and higher data rates are required in a bandwidth to make available all these services. However, multipath propagation phenomenon is still the major challenge factor which is causing frequency selective fading that comes from various echoes of transmitted symbols interference at the end of receiver and thus cause bit error rate (BER) degradation [14].

BER in wireless communications can be caused surely via all types of disturbances. Thus, using channel coding technologies are best choice to minimize the impact of actual channel. Where, BoseChaudhuri-Hocquenghem (BCH) coding considered a good type of linear error correction codes owing to its powerful correction capability as well it is so close to theory value. Furthermore, BCH code is commonly using in data transmission because of it has some effective coding and decoding algorithms because of the strict algebraic architecture. In additional to, the structure of $\mathrm{BCH}$ coding with interleaving contribute in correcting both of burst and random errors and thus greatly reducing BER of the system [15].

Generally, to achieve reliable transmission in digital communication systems, using error correcting codes are considered an essential over noisy channels [16]. Hence, to reduce BER in LTE system via MIMO channel, BCH codes have been suggested in [17]. They showed that using BCH codes that have low decoding complexity is contributed in controlling of errors in LTE system via LTE-MIMO channel. Whilst, simulation results indicated that $\mathrm{BCH}$ codes outperform than both convolutional and turbo codes in reducing BER.

$\mathrm{BCH}$ codes are also proposed in [18] to achieve reliable data transmission of filtered-OFDM system via multi-path fading channel. The results showed that using BCH code with f-OFDM system achieved significant improving in terms of BER performance and OOBE better than conventional OFDM system. Although, PAPR in their system was high due to the tradeoff among OOBE, PAPR and SNR performance. Nevertheless, they indicated that their proposed system is considered a competitor candidate for 5G communication systems to meet its requirements owing to the ability of decreasing both OOBE and BER. So, in this paper, $\mathrm{BCH}$ codes have been proposed to achieve reliable data transmission for UFMC system that have low OOBE and PAPR levels.

\section{SYSTEM MODEL}

Block diagram of using BCH codes with UFMC system over AWGN channel has been described and depicted in Figure 1. The suggested system will be introduced as promising candidate waveform for 5G wireless mobile system because of its ability of solving the OFDM disadvantages. Compared to OFDM, UFMC is dividing the whole band into sub-bands then in each subband, the data will be processing by IDFT and sub-band filter respectively. Lastly, the signals which is filtered in all subbands will be added with each other to create UFMC signal to be ready for transmission [1]. The purpose of using IFFT in UFMC is to protect the subbsand carriers from the interferences. While, by suitable design of filter, using filtering method in UFMC is minimizing the OOBE. Where, filtering the parameterized side lobe attenuation utilized in order to filter the output of IFFT per subband. 
The band filter is used in UFMC to execute the Chebyshev filter operation. In the UFMC receiver the data that comes from the channel will be performed by $3 \mathrm{~N}$ point FFT then adding guard interval of zeros between successive IFFT symbols. Thus, that will protect from inter symbol interference (ISI) that may accrue owing to transmitter filter delay [8]. The same parameters that shown in Table 1, will be used for both OFDM, f-OFDM systems and proposed UFMC system to achieve fairly comparison among them and explain the advantages/disadvantages of each of them.

Table 1. System parameters [8]

\begin{tabular}{cc}
\hline Variable & Power $(\mathrm{kW})$ \\
\hline Number of FFT points & 512 \\
Sub band size & 20 \\
No. of Subbands & 10 \\
Sub band offset & 156 \\
Modulation & $64-\mathrm{QAM}$ \\
Filter length & 43 \\
Side lobe attention & 40 \\
Error Correcting Techniques & $\mathrm{BCH}(15,5)$ Codes \\
\hline
\end{tabular}

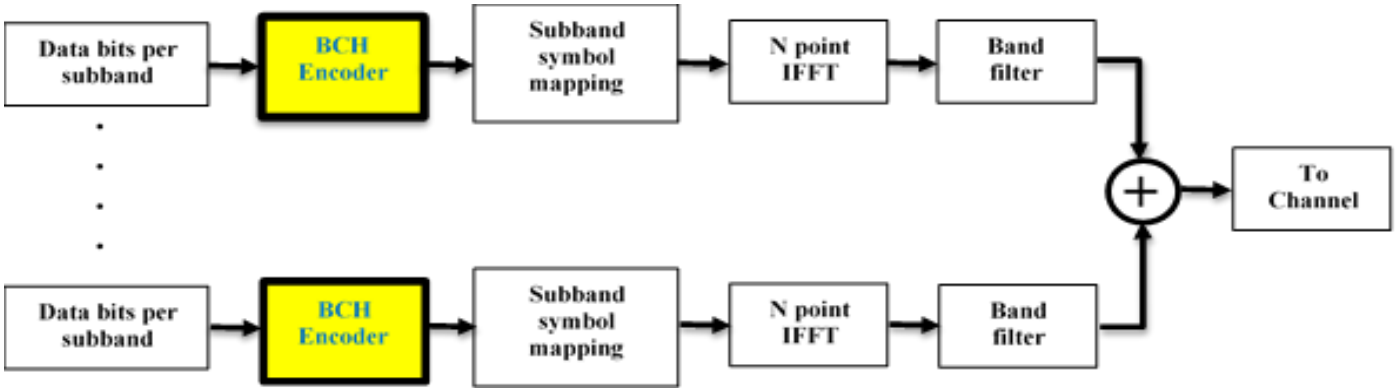

(a)

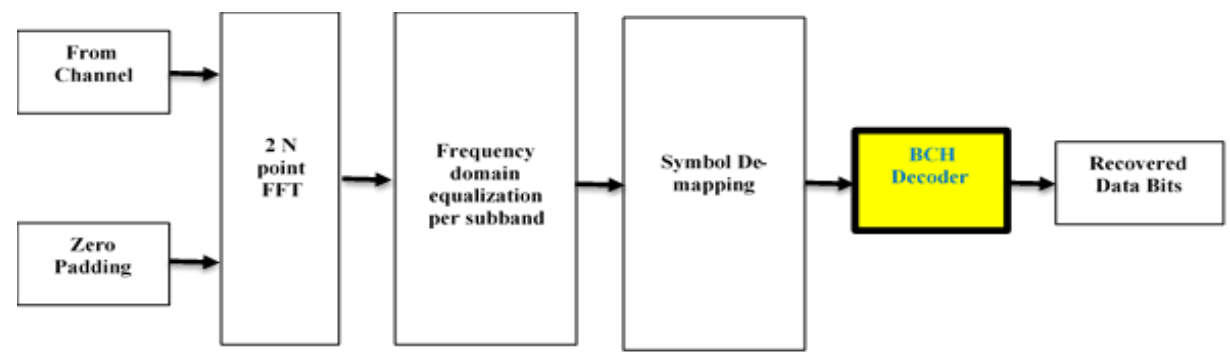

(b)

Figure 1. UFMC block diagram, (a) Transmitter, (b) Reciever [8]

In general, $\mathrm{BCH}$ codes are special type of cyclic code and considered one of the best cyclic codes which have wide applications in storage and communication systems $[19,20]$. Where $\mathrm{BCH}$ codes have been intensively studies because of its strict algebraic structure. The codeword of $\mathrm{BCH}$ code is generated by divide the polynomial $\mathrm{m}(\mathrm{x})$ by a generated polynomial $\mathrm{g}(\mathrm{x})$, then take the rest as parity check bits $\mathrm{r}(\mathrm{x})$ [21]. Hence, by using source encoding, the message will be converted into coded form which are transmitting via channel which is adding extra bits in order using it in detection and correcting errors [22]. So, the encoded data $\mathrm{c}(\mathrm{x})$ has been explained in (1) [21]:

$$
\mathrm{C}(\mathrm{x})=\mathrm{m}(\mathrm{x})+\mathrm{r}(\mathrm{x})
$$

By selected $\mathrm{g}(\mathrm{x})$, the characteristics of the code will be determined. Where, $\mathrm{BCH}$ code could be correct $\leq \mathrm{t}$ independent errors (for integer $\mathrm{t}$ and $\mathrm{m}$ ) [21]:

$$
\begin{aligned}
& n=2^{m}-1 \\
& (n-k) \leq m t \\
& d \geq(2 t+1)
\end{aligned}
$$


where, $\mathrm{n}$ is the block length, $\mathrm{k}$ is code dimension, $(\mathrm{n}-\mathrm{k})$ is parity check bits, $\mathrm{d}$ is minimum hamming distance and $t$ is the capability of error correction $[17,21]$. In this paper, $\mathrm{BCH}(15,5)$ has been used.

\section{RESULTS AND DISCUSSION}

The results in this paper are divided into two stages; BER performance of UFMC system using BCH code over AWGN has been discussed and compared with un-coded system and both OFDM and f-OFDM systems in first stage. In second stage, OOBE and PAPR levels of UFMC have been discussed and compared with conventional OFDM system. Where, the aim of comparing the proposed system with conventional OFDM system which is a core waveform of $4 \mathrm{G}[23,24]$ and f-OFDM system which is a competitor candidate of 5G [25] to show the merits/demerits of proposed system against both of them.

First, Figure 2 shows the comparison of uncoded systems performance for OFDM, f-OFDM and UFMC systems over AWGN channel. It reveals that the performance of both UFMC and OFDM systems are somehow close to one another. While, f-OFDM system outperforms both UFMC and OFDM systems. Thus, the uncoded f-OFDM system performance was the best among of uncoded OFDM and UFMC systems in terms of BER.

In the other hand, the performance of UFMC system using BCH codes through AWGN channel has been showed in Figure 3. It reveals that the performance of proposed system using $\mathrm{BCH}$ code is considerably enhanced better than uncoded system. Thus, using BCH code in UFMC system contributes in enhancing BER performance through decreasing BER, where the errors goes to zero at $12 \mathrm{~dB}$ SNR for proposed system against $20 \mathrm{~dB}$ SNR for uncoded system.

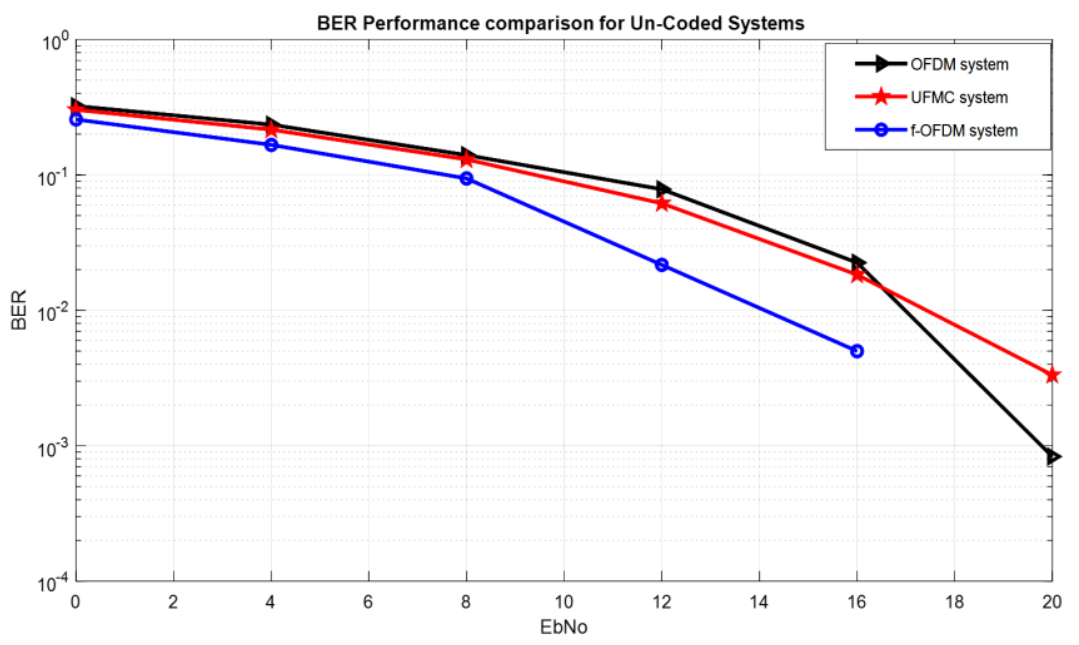

Figure 2. The comparison of uncoded systems performance

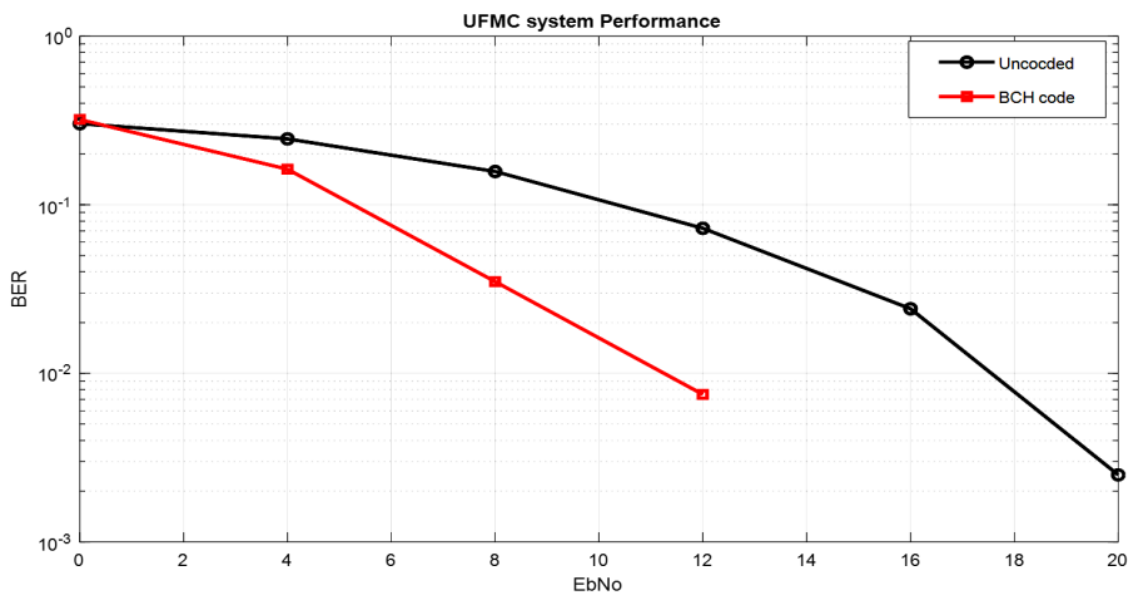

Figure 3. The performance of UFMC system 
Whereas, the comparison of system performance between UFMC and f-OFDM systems using BCH codes is depicted in Figure 4. The result reveals that the performance of f-OFDM system using BCH code still outperforms UFMC system. However, using BCH code in the proposed UFMC system contributed in improving BER performance to be better than uncoded UFMC system as shown in Figure 3.

Second, the comparison of OOBE between OFDM and UFMC systems has been depicted in Figure 5 [8]. It shows that 200 subcarriers for OFDM system while 10 subbands which is divided in whole band for UFMC system. The result shows that the side lobes of UFMC was less than OFDM and thus, OOBE was lower for UFMC system and better than familiar OFDM system. Where, OOBE of UFMC system was around $40 \mathrm{~dB}$ lower than OFDM system. Whereas, the values of PAPR of OFDM versus UFMC systems have been explained in Table 2 .

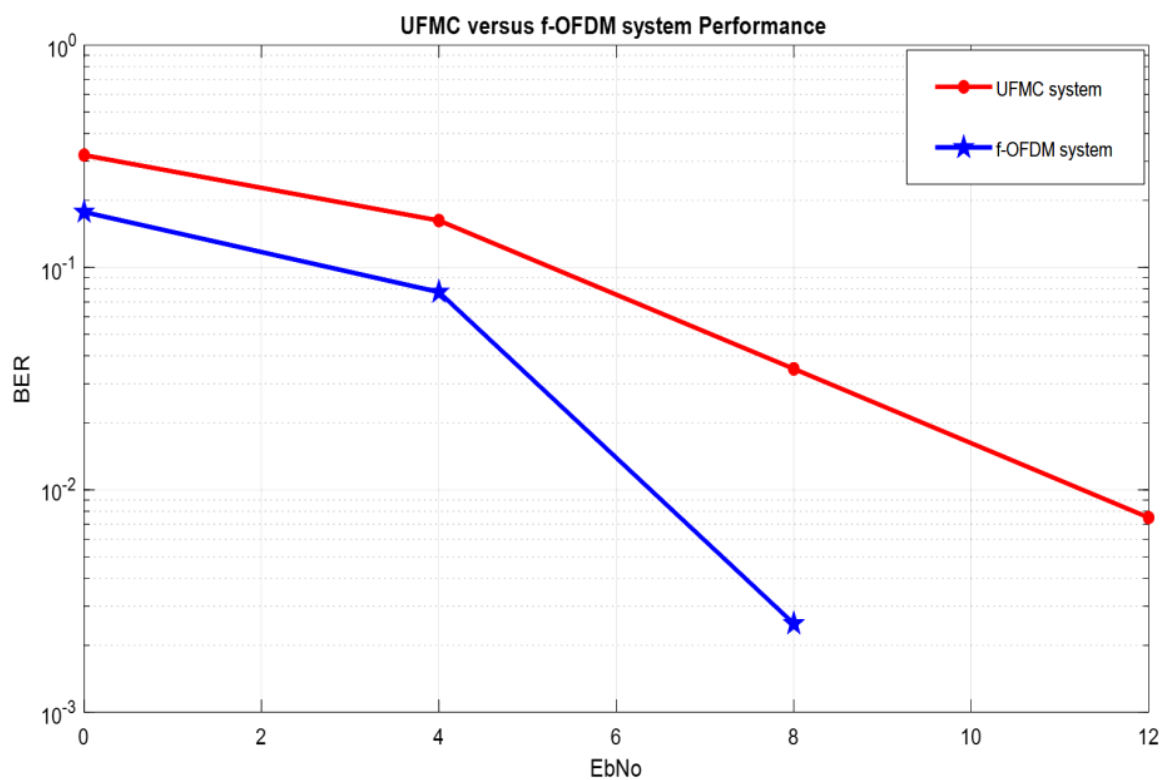

Figure 4. The comparison between UFMC and f-OFDM system performance
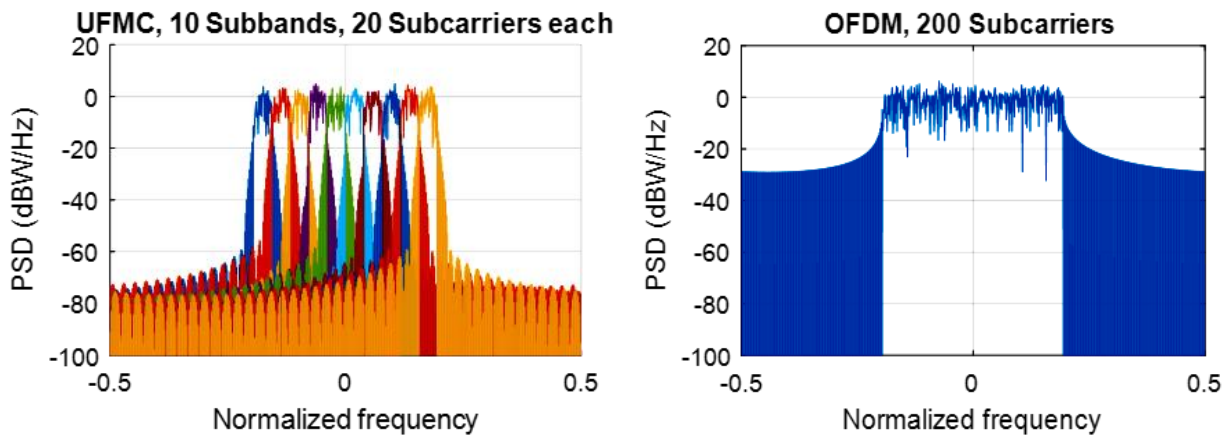

Figure 5. Power spectral density of OFDM vs UFMC

Table 2. (PAPR values)

\begin{tabular}{cccc}
\hline & OFDM & Uncoded UFMC & BCH-UFMC \\
\hline PAPR $(\mathrm{dB})$ & 9.9269 & 8.6229 & 8.6899 \\
\hline
\end{tabular}

The comparison in Table 2 explained that PAPR values of uncoded-UFMC system is lower than conventional OFDM system. Moreover, the suggested UFMC system using BCH codes is also lower than OFDM system. Where, PAPR for OFDM system was $9.9269 \mathrm{~dB}$ while it was $8.6229 \mathrm{~dB}$ and $8.6899 \mathrm{~dB}$ for Uncoded and BCH codes with UFMC system respectively. 
Although, BER performance of f-OFDM system is better than UFMC system in both uncoded and with $\mathrm{BCH}$ codes. While, both uncoded-UFMC and OFDM systems were somehow close to one another and UFMC system performance is enhancing more using $\mathrm{BCH}$ codes. However, the suggested UFMC could be considered a competitor candidate of $5 \mathrm{G}$ wireless communication systems owing to the ability of decreasing both OOBE and PAPR while enhancing BER performance better than conventional OFDM system.

\section{CONCLUSION}

In this paper, UFMC system has been proposed as a solution for disadvantages of OFDM system to be used as a competitor waveform of $5 \mathrm{G}$ wireless communication systems. Where, high both PAPR and OOBE values are considered a common demerits of OFDM systems which are prevent using it for next generation. $\mathrm{BCH}$ codes have been proposed in this paper for UFMC system to improve the BER performance. Whereas, the proposed system has been compared with both OFDM and f-OFDM system. The outcomes showed that the performance of both uncoded OFDM and UFMC systems are somehow close to one another. While, uncoded f-OFDM system outperforms all of uncoded OFDM and UFMC systems. Although, f-OFDM system performance using $\mathrm{BCH}$ codes outperforms of UFMC system. However, using $\mathrm{BCH}$ code in UFMC system contributes in enhancing BER performance through decreasing BER, where the errors go to zero at $12 \mathrm{~dB}$ SNR for proposed system against $20 \mathrm{~dB}$ SNR for uncoded UFMC system. In other hand, the values of PAPR and OOBE for proposed UFMC were lower than conventional OFDM system. Where, PAPR for OFDM system was $9.9269 \mathrm{~dB}$ while it was $8.6229 \mathrm{~dB}$ and $8.6899 \mathrm{~dB}$ for Uncoded and $\mathrm{BCH}$ codes with UFMC system respectively. Furthermore, the OOBE level of UFMC system was around $40 \mathrm{~dB}$ lower than OFDM system. Hence, the proposed UFMC can considered a contender candidate for 5G wireless communication system because of its ability of decreasing each of OOBE and PAPR while enhancing BER performance better than conventional OFDM system.

\section{ACKNOWLEDGEMENTS}

The authors would like to thank the Ministry of Higher Education Malaysia under Fundamental Research Grant Scheme (FRGS/1/2019/TK10/UTHM/02/3) and Universiti Tun Hussein Onn Malaysia for generous financial support.

\section{REFERENCES}

[1] A. F. Almutairi, et al., "Performance Analysis of Hybrid Peak to Average Power Ratio Reduction Techniques in 5G UFMC Systems," IEEE Access, vol. 7, pp. 80651 - 80660, 2019.

[2] M. A. Taher., "Enhanced 5G Throughput using UFMC Multiplexing," Journal of Southwest Jiaotong University, vol. 54, no. 5, pp. 1-11, 2019.

[3] Y. Zhao, et al., "Resource block filtered-OFDM as a multi-carrier transmission scheme for 5G," ScienceDirect, Computers and Electrical Engineering, vol. 72, pp. 543-552, 2018.

[4] A. K. Baki, et al., "Novel Methods of Filtering for FBMC/UFMC Based 5G Communication Systems," 2019 7th International Conference on Smart Computing \& Communications (ICSCC), pp. 1-4, 2019.

[5] I. Baig, et al., "A Low PAPR DHT Precoding Based UFMC Scheme for 5G Communication Systems," 2019 6th International Conference on Control, Decision and Information Technologies (CoDIT), pp. 425-428, 2019.

[6] J. Wang, et al., "Spectral Efficiency Improvement with 5G Technologies: Results from Field Tests," IEEE Journal on Selected Areas in Communications, vol. 35, no. 8, pp. 1867 - 1875, 2017.

[7] Y. Qui, et al., "Filtered bank based implementation for filtered OFDM," 7th IEEE International Conference on Electronics Information and Emergency Communication (ICEIEC), pp. 15-18, 2017.

[8] P. N. Rani, and Ch. S. Rani, "UFMC: The 5G Modulation Technique," 2016 IEEE International Conference on Computational Intelligence and Computing Research (ICCIC), pp. 1-3, 2016.

[9] Gh. A. Hussain and L. Audah, "UFMC system performance improvement using RS codes for 5G communication system," TELKOMNIKA Telecommunication, Computing, Electronics and Control, vol. 18, no. 4, pp. 1843-1848, 2020.

[10] J. Wen, et al., "Design of Waveform Shaping Filter in the UFMC System," IEEE Access, vol. 6, pp. 32300-32309, 2018.

[11] S. Buzzi, et al., "MIMO-UFMC Transceiver Schemes for Millimeter-Wave Wireless Communications," IEEE Transactions on Communications, vol. 67, no. 5, pp. 1-12, 2019.

[12] A. Hammoodi, et al., "Green Coexistence for 5G Waveform Candidates: A Review." IEEE Access, vol. 7, pp. 10103-10126, 2019.

[13] R. Teja and Sh. R. Chopra, "Review of UFMC Technique in 5G," 2018 International Conference on Intelligent Circuits and Systems (ICICS), pp.115-120, 2018. 
[14] S. S. Sarnin, et al., "Performance evaluation of phase shift keying modulation technique using BCH code, Cyclic code and Hamming code through AWGN channel model in communication system," The 3rd International Conference on Information Sciences and Interaction Sciences, pp. 60-65, 2010.

[15] X. Zhi-Yuan NA, et al., "Design and Study on a New BCH Coding and Interleaving Techniques Based on ARM Chip," 2008 4th IEEE International Conference on Circuits and Systems for Communications, pp. 315-318, 2008.

[16] D. Jo, et al., "Blind Reconstruction of BCH Codes Based on Consecutive Roots of Generator Polynomials," IEEE Communications Letters, vol. 22, no. 5, pp. 894-897, 2018.

[17] G. H. A. Hussain and L. Audah, "Downlink LTE System Performance Improvement by Using BCH Codes over LTE-MIMO Channel," International Journal of Integrated Engineering, vol. 18, no. 4, pp. 1843-1848, 2018.

[18] G. H. A. Hussain and L. Audah, "BCH codes for 5G wireless communication systems over multipath fading channel," Indonesian Journal of Electrical Engineering and Computer Science, vol. 17, no. 1, pp. 310-316, 2020.

[19] C. Ding, "Parameters of Several Classes of BCH Codes," IEEE Transactions on Information Theory, vol. 61, no. 10, pp. 5322- 5330, 2005.

[20] Ch. Li, et al., "On Two Classes of Primitive BCH Codes and Some Related Codes," IEEE Transactions on Information Theory, vol. 65, no. 6, pp. 3830- 3840, 2019.

[21] A. Al-Barrak, et al.,"Utilisation of multipath phenomenon to improve the performance of BCH and RS codes," 2016 8th Computer Science and Electronic Engineering (CEEC), pp. 6-11, 2016.

[22] P. Manhasa and M. K. Sonib, "Performance of OFDM System under Different Fading Channels and Coding," Bulletin of Electrical Engineering and Informatics, vol. 6, no. 1, pp. 54-61, 2017.

[23] Y. J. I. A. Yaqoob, et al.," Performance evaluation of video streaming on LTE with coexistence of Wi-Fi signal," Bulletin of Electrical Engineering and Informatics, vol. 8, no. 3, pp. 890-897, 2019.

[24] G. Bachechka, et al., "Comparative analysis of UFMC technology in 5G," 2017 International Siberian Conference on Control and Communications (SIBCON), pp. 1-6, 2017.

[25] M. Saad, et al., "UFMC Transceiver Complexity Reduction," 2018 25th International Conference on Telecommunications (ICT), pp. 295-301, 2018.

\section{BIOGRAPHIES OF AUTHORS}

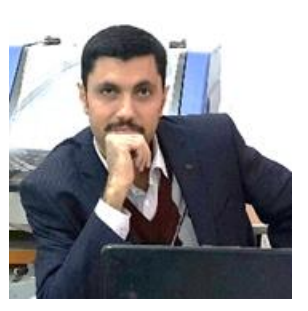

Ghasan A. Hussain is currently a PhD student in Universiti Tun Hussein Onn Malaysia since 2017. He received Bachelor degree in Communication Engineering from Alfatah UniversityTripoli/Libya in 2003. Hussian received the Master degree in communication Engineering from University Putra Malaysia in 2013. Since 2006, he has been with Electrical Engineering, University of Kufa, Iraq, where he is now a lecturer. His research interests include wireless communication systems, Mobile systems, LTE and Error Correction Techniques. He has a number of publications in different journals in his field.

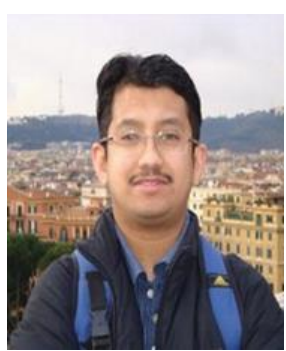

Lukman Audah received his BS degree in electrical engineering from the University Technology Malaysia, Johor, Malaysia, in 2005, and his MS and PhD degrees in communication networks and software from the University of Surrey, United Kingdom, in 2007 and 2013, respectively. Since 2014, he has been with the Faculty of Electrical and Electronic Engineering, University Tun Hussein Onn Malaysia, Johor, Malaysia, where he is now a Senior lecturer. His main research interests are wireless and mobile communications, Internet traffic engineering, network system management, data security, and satellite communications. 